\title{
Boundary value problems for the one-dimensional Willmore equation
}

\author{
Klaus Deckelnick* and Hans-Christoph Grunau ${ }^{\dagger}$ \\ Fakultät für Mathematik \\ Otto-von-Guericke-Universität \\ Postfach 4120 \\ D-39016 Magdeburg
}

November 17, 2006

\begin{abstract}
The one-dimensional Willmore equation is studied under Navier as well as under Dirichlet boundary conditions. We are interested in smooth graph solutions, since for suitable boundary data, we expect the stable solutions to be among these.

In the first part, classical symmetric solutions for symmetric boundary data are studied and closed expressions are deduced. In the Navier case, one has existence of precisely two solutions for boundary data below a suitable threshold, precisely one solution on the threshold and no solution beyond the threshold. This effect reflects that we have a bending point in the corresponding bifurcation diagram and is not due to that we restrict ourselves to graphs. Under Dirichlet boundary conditions we always have existence of precisely one symmetric solution.

In the second part, we consider boundary value problems with nonsymmetric data. Solutions are constructed by rotating and rescaling suitable parts of the graph of an explicit symmetric solution.

One basic observation for the symmetric case can already be found in Euler's work. It is one goal of the present paper to make Euler's observation more accessible and to develop it under the point of view of boundary value problems. Moreover, general existence results are proved.
\end{abstract}

\section{Introduction}

Recently, the Willmore functional and the associate $L^{2}$-gradient flow, the so-called Willmore flow, have attracted a lot of attention. Given a smooth immersed surface $f: M \rightarrow \mathbb{R}^{3}$, the Willmore functional is defined by

$$
W(f):=\int_{f(M)} H^{2} d A
$$

where $H=\left(\kappa_{1}+\kappa_{2}\right) / 2$ denotes the mean curvature of $f(M)$. Apart from being of geometric interest, the functional $W$ is a model for the elastic energy of thin shells or biological membranes. Furthermore, it is used in image processing for problems of surface restoration and image inpainting. In these applications one is usually concerned with minima, or more generally with critical points of the Willmore functional. It is well-known that the corresponding surface $\Gamma$ has to satisfy the Willmore equation

$$
\Delta H+2 H\left(H^{2}-K\right)=0 \quad \text { on } \Gamma,
$$

\footnotetext{
*e-mail: Klaus.Deckelnick@mathematik.uni-magdeburg.de

${ }^{\dagger}$ e-mail: Hans-Christoph.Grunau@mathematik.uni-magdeburg.de
} 
where $\Delta$ denotes the Laplace-Beltrami operator on $\Gamma$ and $K$ its Gauß curvature. A solution of (1) is called a Willmore surface. Existence of closed Willmore surfaces of prescribed genus has been proved by Simon [Sn] and Bauer \& Kuwert [BK]. Also, local and global existence results for the Willmore flow of closed surfaces are available, see e.g. [KS1, KS2, KS3, St]. The Willmore flow for one dimensional closed curves was studied by [P, DKS].

If one is interested in surfaces with boundaries, then appropriate boundary conditions have to be added to (1). Since this equation is of fourth order one requires two sets of conditions and a discussion of possible choices can be found in [Nit] along with corresponding existence results. These results, however, are based on perturbation arguments and hence require severe smallness conditions on the data, which are by no means explicit. Thus the question arises whether it is possible to specify more general conditions on the boundary data that will guarantee the existence of a solution to (1). Such a task seems to be quite difficult since the problem is highly nonlinear and in addition lacks a maximum principle.

In order to gain some insight it is natural to look at the one-dimensional case first, where in some situations, almost explicit solutions can be found for suitable boundary value problems. Critical points of the total squared curvature functional $\int_{\Gamma} \kappa^{2} d s$ are called elastic curves and the analogue of (1) reads

$$
\kappa_{s s}+\frac{1}{2} \kappa^{3}=0 \quad \text { on } \Gamma \text {. }
$$

Here, $s$ denotes arclength of $\Gamma$. In view of the scaling properties of the total squared curvature functional one often considers $\int_{\Gamma}\left(\kappa^{2}+\lambda\right) d s$, at least in the case of closed curves leading to an additional term $\lambda \kappa$ in (2). It is possible to describe the solutions of (2) in terms of elliptic integrals. Many results and closed parametric expressions for solutions can already be found in the "Additamentum, De Curvis Elasticis" in Vol. 24 of the first series of Euler's "Opera omnia" [E, pp. 231-297], cf. also [Nit, pp. 381-383]. More recently, Langer and Singer [LS] gave explicit descriptions for closed curves in manifolds of constant sectional curvature. Formulae for non-closed curves in the plane are derived in $[\mathrm{LI}]$. Depending on the conditions prescribed at the fixed endpoints, a nonlinear system of up to three equations needs to be solved in order to determine the parameters that appear in the elliptic integrals.

In the above papers the solutions are given in terms of arclength parametrizations on a-priori unknown parameter domains. In the present work we take a different point of view: the curves under consideration are given as graphs over a fixed domain which we take for simplicity as the unit interval $[0,1]$. In this case the Willmore functional becomes

$$
W(u)=\int_{\operatorname{graph}(u)} \kappa(x)^{2} d s(x)=\int_{0}^{1} \kappa(x)^{2} \sqrt{1+u^{\prime}(x)^{2}} d x,
$$

where

$$
\kappa(x)=\frac{d}{d x}\left(\frac{u^{\prime}(x)}{\sqrt{1+u^{\prime}(x)^{2}}}\right)=\frac{u^{\prime \prime}(x)}{\left(1+u^{\prime}(x)^{2}\right)^{3 / 2}}
$$

is the curvature of the graph of $u$ at the point $(x, u(x))$. The Willmore equation now takes the form

$$
\frac{1}{\sqrt{1+u^{\prime}(x)^{2}}} \frac{d}{d x}\left(\frac{\kappa^{\prime}(x)}{\sqrt{1+u^{\prime}(x)^{2}}}\right)+\frac{1}{2} \kappa^{3}(x)=0, \quad x \in(0,1),
$$

see Lemma 2 below.

We shall focus on two different sets of boundary conditions. Firstly, for given values $\alpha_{0}, \alpha_{1} \in \mathbb{R}$ we shall consider Navier boundary conditions

$$
u(0)=u(1)=0, \quad \kappa(0)=-\alpha_{0}, \kappa(1)=-\alpha_{1} .
$$


In the first part of the paper we confine ourselves to the case of symmetric boundary conditions $\alpha_{0}=\alpha_{1}=\alpha$. Note that critical points of $(3)$ in $H^{2}(0,1) \cap H_{0}^{1}(0,1)$ will satisfy $\kappa(0)=\kappa(1)=0$ as natural boundary conditions. In order to obtain the corresponding property for nonzero $\alpha$ one has to replace $W$ by the functional

$$
\tilde{W}_{\alpha}(u)=\int_{\operatorname{graph}(u)}\left(\kappa(x)^{2}+2 \alpha \kappa(x)\right) d s(x)=\int_{0}^{1}\left(\kappa(x)^{2}+2 \alpha \kappa(x)\right) \sqrt{1+u^{\prime}(x)^{2}} d x,
$$

see Corollary 1 below. As a second set of boundary conditions we shall examine Dirichlet boundary conditions

$$
u(0)=u(1)=0, \quad u^{\prime}(0)=\beta_{0}, u^{\prime}(1)=-\beta_{1},
$$

where $\beta_{0}$ and $\beta_{1}$ are real parameters. Again, we first focus on the symmetric situation, while nonsymmetric solutions are discussed in Sect. 6 .

Note that due to the presence of the length element $\sqrt{1+u^{\prime}(x)^{2}}$ the nice structure of $(2)$ is lost. Nevertheless it is still possible to obtain formulae for the solutions of the above boundary value problems. The key observation in deriving these expressions is that if $u$ is a solution of (5), then the auxiliary function

$$
v(x):=\kappa(x)\left(1+u^{\prime}(x)^{2}\right)^{1 / 4}
$$

satisfies a second order differential equation of the form

$$
-\left(a(x) v^{\prime}(x)\right)^{\prime}+b(x) v^{\prime}(x)=0 .
$$

Here, the coefficients $a(x), b(x)$ depend on the solution $u$. A similar observation was already made by Euler, see [E, p. 234, line 13]. In the case of a symmetric solution we shall be able to conclude that $v$ is a constant, more precisely we have the following result:

Lemma 1. Let $u \in C^{4}([0,1])$ be a function being symmetric around $x=1 / 2$ and define

$$
c_{0}:=\int_{\mathbb{R}} \frac{1}{\left(1+\tau^{2}\right)^{5 / 4}} d \tau=\mathcal{B}\left(\frac{1}{2}, \frac{3}{4}\right)=\sqrt{\pi} \frac{\Gamma(3 / 4)}{\Gamma(5 / 4)}=2.396280469 \ldots
$$

Then $u$ solves the Willmore equation (5) iff there exists a constant $c \in\left(-c_{0}, c_{0}\right)$ such that

$$
\forall x \in[0,1]: \quad \kappa(x)\left(1+u^{\prime}(x)^{2}\right)^{1 / 4}=-c .
$$

Having found the above representation we can then examine the abovementioned boundary value problems. Solvability of the Navier boundary value problem in the case of symmetric data strongly depends on whether the boundary datum $|\alpha|$ is below a threshold $\alpha_{\max }$ or not.

Theorem 1. There exists $\alpha_{\max }=1.343799725 \ldots$ such that for $0<|\alpha|<\alpha_{\max }$, the Navier boundary value problem

$$
\left\{\begin{array}{c}
\frac{1}{\sqrt{1+u^{\prime}(x)^{2}}} \frac{d}{d x}\left(\frac{\kappa^{\prime}(x)}{\sqrt{1+u^{\prime}(x)^{2}}}\right)+\frac{1}{2} \kappa^{3}(x)=0, \quad x \in(0,1), \\
u(0)=u(1)=0, \quad \kappa(0)=\kappa(1)=-\alpha
\end{array}\right.
$$

has precisely two smooth (graph) solutions $u$ in the class of smooth functions that are symmetric around $x=\frac{1}{2}$. If $|\alpha|=\alpha_{\max }$ one has precisely one such solution, for $\alpha=0$ one only has the trivial solution and no such solutions exist for $|\alpha|>\alpha_{\max }$. 

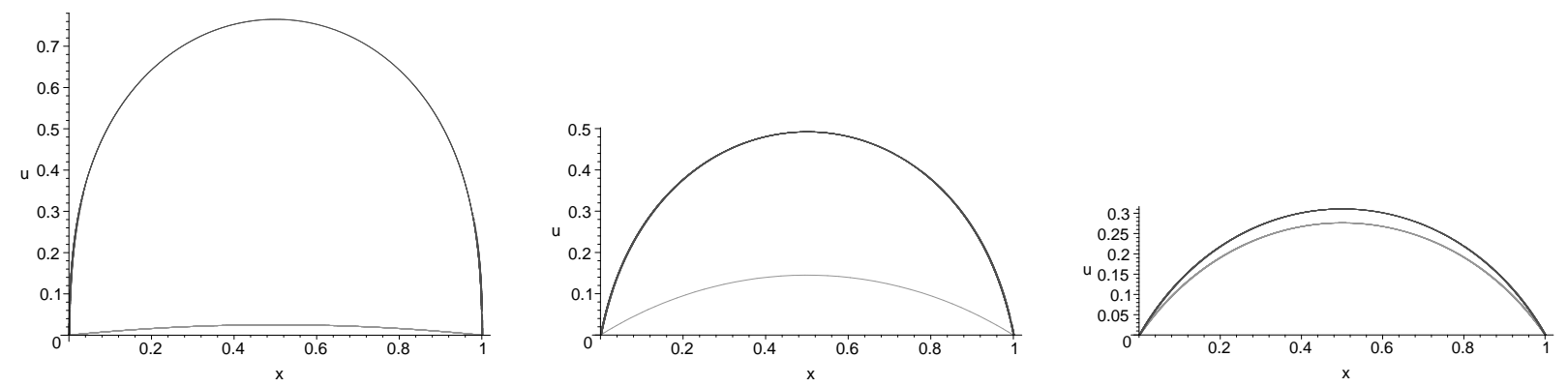

Figure 1: Solutions of the Navier boundary value problem (11) for $\alpha=0.2, \alpha=1$ and $\alpha=1.34$ (left to right)


Figure 2: Bifurcation diagram for (11): The extremals value of the solution $u(1 / 2)$ (left) and of the derivative $u^{\prime}(0)$ (right) plotted over $\alpha$

The small solutions are ordered with respect to $\alpha$ while the large ones become smaller for increasing $\alpha$, see Figure 1. For the bifurcation diagram, see Figure 2.

For the Dirichlet problem with symmetric data the situation is - somehow surprisingly - simpler:

Theorem 2. For every $\beta \in \mathbb{R}$, the Dirichlet boundary value problem

$$
\left\{\begin{array}{c}
\frac{1}{\sqrt{1+u^{\prime}(x)^{2}}} \frac{d}{d x}\left(\frac{\kappa^{\prime}(x)}{\sqrt{1+u^{\prime}(x)^{2}}}\right)+\frac{1}{2} \kappa^{3}(x)=0, \quad x \in(0,1), \\
u(0)=u(1)=0, \quad u^{\prime}(0)=-u^{\prime}(1)=\beta
\end{array}\right.
$$

has precisely one smooth (graph) solution $u$ in the class of smooth functions that are symmetric around $x=\frac{1}{2}$. This solution is the unique minimum of the Willmore functional in the class $M_{\beta}:=\left\{v \in H^{2}(0,1) \cap H_{0}^{1}(0,1) \mid v^{\prime}(0)=-v^{\prime}(1)=\beta\right\}$.

The solutions are ordered with respect to $\beta$, cf. Lemma 5 . This means that we have a comparison principle for the Dirichlet problem (12). For the bifurcation diagram, see Figure 3.

Looking for corresponding results for nonsymmetric boundary value problems is more involved and less explicit than in the symmetric situation, although the explicit formulae from the latter 


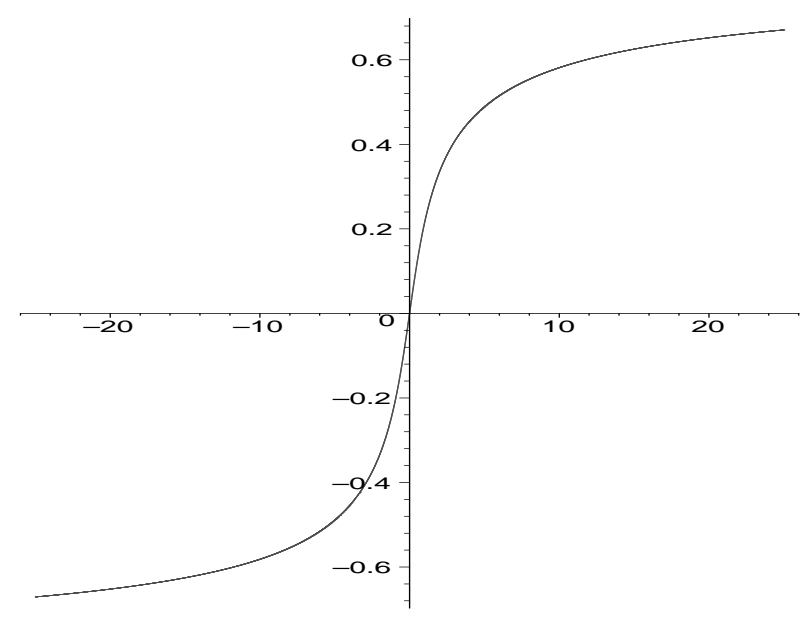

Figure 3: Bifurcation diagram for (12): The extremal value of the solution $u(1 / 2)$ plotted over $\beta$

case are intensively exploited. For the formulation and proofs of such results, which we think are nevertheless somehow exhaustive, we refer to $\S 6$.

This paper is organized as follows. In $\S 2$ we shall consider the Euler-Lagrange equations for $W$ and $\tilde{W}_{\alpha} . \S 3$ is devoted to the derivation of (9) and to provide a proof of Lemma 1 . This result is subsequently used in $\S 4$ to obtain a representation for the solution $u$ itself from which we can easily deduce several qualitative properties. In $\S 5$, we present the proofs of Theorems 1 and 2 . Finally, $\S 6$ is concerned with nonsymmetric boundary value problems.

\section{Euler-Lagrange equation}

For the reader's convenience, we calculate the first variation of the Willmore functional.

Lemma 2. Let $u \in C^{4}([0,1])$ and $\kappa$ denote the corresponding curvature. Then, for all $\varphi \in$ $C_{0}^{\infty}(0,1)$, we have

$$
\left.\frac{d}{d t} W(u+t \varphi)\right|_{t=0}=\int_{0}^{1}\left(\frac{2}{\sqrt{1+u^{\prime}(x)^{2}}} \frac{d}{d x}\left(\frac{\kappa^{\prime}(x)}{\sqrt{1+u^{\prime}(x)^{2}}}\right)+\kappa^{3}(x)\right) \varphi(x) d x .
$$

Proof. We assume only that $\varphi(0)=\varphi(1)=0$.

$$
\begin{aligned}
\left.\frac{d}{d t} W(u+t \varphi)\right|_{t=0}=\frac{d}{d t}\left(\int_{0}^{1} \frac{\left(u^{\prime \prime}(x)+t \varphi^{\prime \prime}(x)\right)^{2}}{\left(1+\left(u^{\prime}(x)+t \varphi^{\prime}(x)\right)^{2}\right)^{5 / 2}} d x\right)_{\mid t=0} \\
=2 \int_{0}^{1} \kappa(x) \frac{\varphi^{\prime \prime}(x)}{1+u^{\prime}(x)^{2}} d x-5 \int_{0}^{1} \kappa(x)^{2} \frac{u^{\prime}(x)}{\sqrt{1+u^{\prime}(x)^{2}}} \varphi^{\prime}(x) d x \\
=2\left[\frac{\kappa(x) \varphi^{\prime}(x)}{1+u^{\prime}(x)^{2}}\right]_{0}^{1}-2 \int_{0}^{1} \kappa^{\prime}(x) \frac{\varphi^{\prime}(x)}{1+u^{\prime}(x)^{2}} d x+4 \int_{0}^{1} \kappa(x) \varphi^{\prime}(x) \frac{u^{\prime}(x) u^{\prime \prime}(x)}{\left(1+u^{\prime}(x)^{2}\right)^{2}} \\
+5 \int_{0}^{1} \kappa(x)^{3} \varphi(x) d x+10 \int_{0}^{1} \kappa(x) \kappa^{\prime}(x) \frac{u^{\prime}(x)}{\sqrt{1+u^{\prime}(x)^{2}}} \varphi(x) d x
\end{aligned}
$$




$$
\begin{aligned}
= & 2\left[\frac{\kappa(x) \varphi^{\prime}(x)}{1+u^{\prime}(x)^{2}}\right]_{0}^{1}+2 \int_{0}^{1}\left(\frac{\kappa^{\prime}(x)}{\sqrt{1+u^{\prime}(x)^{2}}}\right)^{\prime} \frac{\varphi(x)}{\sqrt{1+u^{\prime}(x)^{2}}} \\
& -2 \int_{0}^{1} \frac{\kappa^{\prime}(x)}{\sqrt{1+u^{\prime}(x)^{2}}} \varphi(x) \frac{u^{\prime}(x) u^{\prime \prime}(x)}{\left(1+u^{\prime}(x)^{2}\right)^{3 / 2}}+4 \int_{0}^{1} \kappa(x)^{2} \varphi^{\prime}(x) \frac{u^{\prime}(x)}{\sqrt{1+u^{\prime}(x)^{2}}} d x \\
& +5 \int_{0}^{1} \kappa(x)^{3} \varphi(x) d x+10 \int_{0}^{1} \kappa(x) \kappa^{\prime}(x) \frac{u^{\prime}(x)}{\sqrt{1+u^{\prime}(x)^{2}}} \varphi(x) d x \\
= & 2\left[\frac{\kappa(x) \varphi^{\prime}(x)}{1+u^{\prime}(x)^{2}}\right]_{0}^{1}+2 \int_{0}^{1} \frac{1}{\sqrt{1+u^{\prime}(x)^{2}}}\left(\frac{\kappa^{\prime}(x)}{\sqrt{1+u^{\prime}(x)^{2}}}\right)^{\prime} \varphi(x) d x \\
+ & 8 \int_{0}^{1} \kappa(x) \kappa^{\prime}(x) \frac{u^{\prime}(x)}{\sqrt{1+u^{\prime}(x)^{2}}} \varphi(x) d x-8 \int_{0}^{1} \kappa(x) \kappa^{\prime}(x) \frac{u^{\prime}(x)}{\sqrt{1+u^{\prime}(x)^{2}}} \varphi(x) d x \\
& -4 \int_{0}^{1} \kappa(x)^{3} \varphi(x) d x+5 \int_{0}^{1} \kappa(x)^{3} \varphi(x) d x \\
= & 2\left[\frac{\kappa(x) \varphi^{\prime}(x)}{1+u^{\prime}(x)^{2}}\right]_{0}^{1}+2 \int_{0}^{1} \frac{1}{\sqrt{1+u^{\prime}(x)^{2}}}\left(\frac{\kappa^{\prime}(x)}{\sqrt{1+u^{\prime}(x)^{2}}}\right)^{\prime} \varphi(x) d x+\int_{0}^{1} \kappa(x)^{3} \varphi(x) d x .
\end{aligned}
$$

Corollary 1 Let $u \in C^{4}([0,1]) \cap H_{0}^{1}(0,1)$ and $\kappa$ denote the corresponding curvature. We assume that $u$ is a critical point of the modified Willmore functional $\tilde{W}_{\alpha}$ for some fixed $\alpha \in \mathbb{R}$. So for all $\varphi \in C^{\infty}[0,1]$ with $\varphi(0)=\varphi(1)=0$ one has

$$
\frac{d}{d t} \tilde{W}_{\alpha}(u+t \varphi)_{\mid t=0}=0 .
$$

Then $u$ is a solution of the Willmore equation (5), subject to the Navier boundary conditions:

$$
u(0)=u(1)=0, \quad \kappa(0)=\kappa(1)=-\alpha .
$$

Proof. It remains to calculate

$\frac{d}{d t}\left(\int_{0}^{1} \frac{u^{\prime \prime}(x)+t \varphi^{\prime \prime}(x)}{1+\left(u^{\prime}(x)+t \varphi^{\prime}(x)\right)^{2}} d x\right)_{\mid t=0}=\int_{0}^{1}\left(\frac{\varphi^{\prime \prime}(x)}{1+u^{\prime}(x)^{2}}-2 \frac{u^{\prime \prime}(x) u^{\prime}(x) \varphi^{\prime}(x)}{\left(1+u^{\prime}(x)^{2}\right)^{2}}\right) d x=\left[\frac{\varphi^{\prime}(x)}{1+u^{\prime}(x)^{2}}\right]_{0}^{1}$.

Combining this result with Lemma 2 , we obtain

$$
\begin{aligned}
0 & =\frac{d}{d t} \tilde{W}_{\alpha}(u+t \varphi)_{\mid t=0} \\
& =2\left[\frac{(\kappa(x)+\alpha) \varphi^{\prime}(x)}{1+u^{\prime}(x)^{2}}\right]_{0}^{1}+2 \int_{0}^{1} \frac{1}{\sqrt{1+u^{\prime}(x)^{2}}}\left(\frac{\kappa^{\prime}(x)}{\sqrt{1+u^{\prime}(x)^{2}}}\right)^{\prime} \varphi(x) d x+\int_{0}^{1} \kappa(x)^{3} \varphi(x) d x .
\end{aligned}
$$

Taking first arbitrary $\varphi \in C_{0}^{\infty}(0,1)$, we see that $u$ solves the Euler-Lagrange equation (5) and that for all $\varphi \in C^{\infty}[0,1]$ with $\varphi(0)=\varphi(1)=0$ :

$$
\left[\frac{(\kappa(x)+\alpha) \varphi^{\prime}(x)}{1+u^{\prime}(x)^{2}}\right]_{0}^{1}=0 .
$$

This implies

$$
\kappa(0)=\kappa(1)=-\alpha
$$




\section{The differential equation for the auxiliary function $v$}

We assume that $u \in C^{4}([0,1])$ solves the Willmore equation (5) and recall the definition of the fundamental auxiliary function

$$
v(x):=\kappa(x)\left(1+u^{\prime}(x)^{2}\right)^{1 / 4} .
$$

The crucial point is that $v$ satisfies a second order differential equation without term of order zero, see also [E, pp. 233-234].

Lemma 3. For $x \in[0,1]$ we have:

$$
-\frac{d}{d x}\left(\left(1+u^{\prime}(x)^{2}\right)^{-3 / 4} v^{\prime}(x)\right)+\frac{\kappa(x) u^{\prime}(x)}{\left(1+u^{\prime}(x)^{2}\right)^{1 / 4}} v^{\prime}(x)=0 .
$$

Proof. By straightforward calculations we obtain:

$$
\begin{aligned}
v^{\prime}(x) & =\kappa^{\prime}(x)\left(1+u^{\prime}(x)^{2}\right)^{1 / 4}+\frac{1}{2} \kappa(x)^{2} u^{\prime}(x)\left(1+u^{\prime}(x)^{2}\right)^{3 / 4} ; \\
\left(1+u^{\prime}(x)^{2}\right)^{-3 / 4} v^{\prime}(x) & =\frac{\kappa^{\prime}(x)}{\sqrt{1+u^{\prime}(x)^{2}}}+\frac{1}{2} \kappa(x)^{2} u^{\prime}(x) .
\end{aligned}
$$

By making use of the Willmore equation (5) we conclude:

$$
\begin{aligned}
& \frac{d}{d x}\left(\left(1+u^{\prime}(x)^{2}\right)^{-3 / 4} v^{\prime}(x)\right)=\frac{d}{d x}\left(\frac{\kappa^{\prime}(x)}{\sqrt{1+u^{\prime}(x)^{2}}}\right)+\frac{1}{2} \kappa(x)^{2} u^{\prime \prime}(x)+\kappa(x) \kappa^{\prime}(x) u^{\prime}(x) \\
& \quad=\frac{1}{2} \kappa(x)^{3} u^{\prime}(x)^{2} \sqrt{1+u^{\prime}(x)^{2}}+\kappa(x) \kappa^{\prime}(x) u^{\prime}(x)=\frac{\kappa(x) u^{\prime}(x)}{\left(1+u^{\prime}(x)^{2}\right)^{1 / 4}} v^{\prime}(x)
\end{aligned}
$$

Corollary 2. By the preceding lemma we know that $v$ satisfies a maximum principle. In particular we may conclude for any solution $u$ of the Willmore equation (5): If $\kappa(0), \kappa(1)<0$, then $\kappa<0$ in $[0,1]$. If $\kappa(0)=\kappa(1)=0$, then $\kappa=0$ in $[0,1]$ and the solution $u$ is a straight line segment. If we additionally assume $u(0)=u(1)=0$ then $u(x) \equiv 0$ in $[0,1]$. That means that we have uniqueness for the homogeneous Navier boundary value problem (11) in the class of smooth graphs without assuming a priori any smallness on the solution.

Proof of Lemma 1. Let again $v(x):=\kappa(x)\left(1+u^{\prime}(x)^{2}\right)^{1 / 4}$.

In order to prove necessity of condition (10), we observe first that $v(0)=v(1)$ by our symmetry assumption on $u$. Since $v$ solves a second order (linearized) differential equation without term of order zero, we conclude that there exists $c \in \mathbb{R}$ such that $\forall x \in[0,1]: v(x)=-c$. The additional statement on the admissible range $c \in\left(-c_{0}, c_{0}\right)$ follows from Lemma 4 below.

For proving sufficiency, we start with (10)

$$
\kappa(x)=-\frac{c}{\left(1+u^{\prime}(x)^{2}\right)^{1 / 4}}
$$


and obtain by differentiating

$$
\begin{aligned}
\kappa^{\prime}(x) & =\frac{c}{2} \kappa(x) u^{\prime}(x)\left(1+u^{\prime}(x)^{2}\right)^{1 / 4}=-\frac{1}{2} \kappa(x)^{2} u^{\prime}(x) \sqrt{1+u^{\prime}(x)^{2}} \\
\frac{1}{\sqrt{1+u^{\prime}(x)^{2}}}\left(\frac{\kappa^{\prime}(x)}{\sqrt{1+u^{\prime}(x)^{2}}}\right)^{\prime} & =-u^{\prime}(x) \kappa(x) \frac{\kappa^{\prime}(x)}{\sqrt{1+u^{\prime}(x)^{2}}}-\frac{1}{2} \kappa(x)^{2} \frac{u^{\prime \prime}(x)}{\sqrt{1+u^{\prime}(x)^{2}}} \\
& =\frac{1}{2} u^{\prime}(x)^{2} \kappa(x)^{3}-\frac{1}{2} \kappa(x)^{3}\left(1+u^{\prime}(x)^{2}\right)=-\frac{1}{2} \kappa(x)^{3}
\end{aligned}
$$

so that the Willmore equation (5) is satisfied.

\section{Explicit form of symmetric solutions of the Willmore equation}

In what follows, the function

$$
G: \mathbb{R} \rightarrow\left(-\frac{c_{0}}{2}, \frac{c_{0}}{2}\right), \quad G(s):=\int_{0}^{s} \frac{1}{\left(1+\tau^{2}\right)^{5 / 4}} d \tau
$$

plays a crucial role. It is straightforward to see that $G$ is strictly increasing, bijective with $G^{\prime}(s)>0$. So, also the inverse function

$$
G^{-1}:\left(-\frac{c_{0}}{2}, \frac{c_{0}}{2}\right) \rightarrow \mathbb{R}
$$

is strictly increasing, bijective and smooth with $G^{-1}(0)=0$.

Lemma 4. Let $u \in C^{4}([0,1])$ be a function symmetric around $x=1 / 2$. Then $u$ solves the Willmore equation (5) iff there exists $c \in\left(-c_{0}, c_{0}\right)$ such that

$$
\forall x \in[0,1]: \quad u^{\prime}(x)=G^{-1}\left(\frac{c}{2}-c x\right) .
$$

For the curvature, one has that

$$
\kappa(x)=-\frac{c}{\sqrt[4]{1+G^{-1}\left(\frac{c}{2}-c x\right)^{2}}} .
$$

Moreover, if we additionally assume that $u(0)=u(1)=0$, then one has

$$
u(x)=\frac{2}{c \sqrt[4]{1+G^{-1}\left(\frac{c}{2}-c x\right)^{2}}}-\frac{2}{c \sqrt[4]{1+G^{-1}\left(\frac{c}{2}\right)^{2}}} \quad(c \neq 0) .
$$

Proof. First we show the necessity of the representation formula (17). Let $u \in C^{4}([0,1])$ be a symmetric solution of (5). By Lemma 1 we know that there exists a constant $c \in \mathbb{R}$ such that for all $x \in[0,1]:$

$$
-c=\frac{u^{\prime \prime}(x)}{\left(1+u^{\prime}(x)^{2}\right)^{5 / 4}} .
$$

Integration yields

$$
-c\left(x-\frac{1}{2}\right)=\int_{1 / 2}^{x} \frac{u^{\prime \prime}(\xi)}{\left(1+u^{\prime}(\xi)^{2}\right)^{5 / 4}} d \xi=\int_{0}^{u^{\prime}(x)} \frac{d \tau}{\left(1+\tau^{2}\right)^{5 / 4}}=G\left(u^{\prime}(x)\right) .
$$


Now it becomes apparent that necessarily $(-|c| / 2,|c| / 2) \subset G(\mathbb{R})$ so that $|c|<\int_{\mathbb{R}} \frac{d \tau}{\left(1+\tau^{2}\right)^{5 / 4}}=c_{0}$.

The sufficiency of (17) is obtained by direct calculation and with the help of Lemma 1.

Formula (18) follows by differentiating (17), while for (19) we perform several changes of variables:

$$
\begin{aligned}
u(x) & =\int_{0}^{x} G^{-1}\left(\frac{c}{2}-c s\right) d s=\frac{1}{c} \int_{c / 2-c x}^{c / 2} G^{-1}(\sigma) d \sigma \\
& =\frac{1}{c} \int_{G^{-1}(c / 2-c x)}^{G^{-1}(c / 2)} \frac{t}{\left(1+t^{2}\right)^{5 / 4}} d t=\frac{2}{c \sqrt[4]{1+G^{-1}\left(\frac{c}{2}-c x\right)^{2}}}-\frac{2}{c \sqrt[4]{1+G^{-1}\left(\frac{c}{2}\right)^{2}}}
\end{aligned}
$$

The explicit formulae of the preceding lemma allow for precise statements on the qualitative behaviour of solutions:

Corollary 3. Let $u$ be a smooth solution in $[0,1]$ of the Willmore equation (5), being symmetric around $x=1 / 2$. For any nonconstant solution we have that $\kappa$ is of fixed sign,

$$
\forall x \in(0,1): \quad|\kappa(x)|>|\kappa(0)|,
$$

$x \mapsto u^{\prime}(x)$ is either strictly decreasing or strictly increasing. The following bound for $u$ is independent of $c$ and is valid for all symmetric solutions of the boundary value problems (11), (12), independent of the data $\alpha$ or $\beta$ :

$$
\forall x \in[0,1]: \quad|u(x)|<\frac{2}{c_{0}}=\frac{\sqrt{2 \pi}}{2 \Gamma(3 / 4)^{2}}=0.8346268420 \ldots
$$

Proof. Only the last statement requires a proof. It suffices to show that for $c \in\left(0, c_{0}\right)$

$$
0 \leq u\left(\frac{1}{2}\right)=\frac{2}{c}-\frac{2}{c \sqrt[4]{1+G^{-1}\left(\frac{c}{2}\right)^{2}}}<\frac{2}{c_{0}} .
$$

By changing the variable $c=2 G(d)$, this is equivalent to showing for all $d \in(0, \infty)$ that

$$
H(d):=\frac{2}{c_{0}} G(d)+\left(1+d^{2}\right)^{-1 / 4}-1>0 .
$$

We have $H(0)=0, \lim _{d \rightarrow \infty} H(d)=0$ and

$$
H^{\prime}(d)=\left(\frac{2}{c_{0}}-\frac{d}{2}\right) \frac{1}{\left(1+d^{2}\right)^{5 / 4}} .
$$

This shows that $H$ is increasing first and then decreasing so that $\forall d \in(0, \infty): H(d)>0$.

Moreover, from Lemma 4, we obtain that the smooth symmetric solutions are ordered with respect to $c \in\left(-c_{0}, c_{0}\right)$ and hence with respect to $\beta=u^{\prime}(0)=G^{-1}(c / 2) \in \mathbb{R}$ :

Lemma 5. For the solutions $u=u_{c}$ in Lemma 4, we have that for $c \in\left(-c_{0}, c_{0}\right)$

$$
\forall x \in(0,1): \quad \frac{\partial}{\partial c} u_{c}(x)>0 .
$$

Proof. We obtain from the proof of Lemma 4

$$
\frac{\partial}{\partial c} u(x)=\int_{0}^{x} \frac{\partial}{\partial c} G^{-1}\left(\frac{c}{2}-c s\right) d s=\int_{0}^{x}\left(\frac{1}{2}-s\right)\left(1+G^{-1}\left(\frac{c}{2}-c s\right)^{2}\right)^{5 / 4} d s>0,
$$

since the integrand is odd with respect to $s=1 / 2$, positive first and negative then. 


\section{Boundary value problems}

\section{The Navier boundary value problem}

We obtain all smooth solutions $u=u_{c}$ to (5) being symmetric around $1 / 2$ and satisfying $u_{c}(0)=$ $u_{c}(1)=0$ by formulae (19), (17), (18). This family is parametrised over $c \in\left(-c_{0}, c_{0}\right)$. It remains to consider the dependence of

$$
\alpha=-\kappa_{c}(0)=\frac{c}{\sqrt[4]{1+G^{-1}\left(\frac{c}{2}\right)^{2}}}
$$

on $c$. For this purpose, it is enough to study the function

$$
h:\left(-c_{0}, c_{0}\right) \rightarrow \mathbb{R}, \quad h(c)=\frac{c}{\sqrt[4]{1+G^{-1}\left(\frac{c}{2}\right)^{2}}} .
$$

The range of $h$ is precisely the set of $\alpha$, for which the Navier boundary value problem (11) has a solution. The number of solutions $c$ of the equation $\alpha=h(c)$ is the number of symmetric solutions of the boundary value problem.

Lemma 6. We have $h>0$ in $\left(0, c_{0}\right), h<0$ in $\left(-c_{0}, 0\right), \lim _{c / c_{0}} h(c)=\lim _{c \backslash-c_{0}} h(c)=0$. The function $h$ is odd and has precisely one local maximum in $c_{\max }=1.840428142 \ldots$ and one local minimum in $c_{\min }=-c_{\max }$. The corresponding value is $\alpha_{\max }=h\left(c_{\max }\right)=1.343799725 \ldots$

Proof. First of all we observe that

$$
\lim _{c \nearrow c_{0}} h(c)=-\lim _{c \searrow-c_{0}} h(c)=\frac{c_{0}}{\sqrt[4]{1+\lim _{c / c c_{0}} G^{-1}\left(\frac{c}{2}\right)^{2}}}=0
$$

by definition of $G(\infty)=c_{0} / 2$. Secondly we calculate

$$
\begin{gathered}
h^{\prime}(c)=\frac{1}{\sqrt[4]{1+G^{-1}\left(\frac{c}{2}\right)^{2}}}-\frac{c}{4} G^{-1}\left(\frac{c}{2}\right), \\
h^{\prime \prime}(c)=-\frac{1}{4} G^{-1}\left(\frac{c}{2}\right)-\frac{c}{8}\left(1+G^{-1}\left(\frac{c}{2}\right)^{2}\right)^{5 / 4}<0 \text { in }\left(0, c_{0}\right),
\end{gathered}
$$

so that $h$ is strictly convex in $\left(-c_{0}, 0\right)$ and strictly concave in $\left(0, c_{0}\right)$. This shows that there exists precisely one local maximum $c_{\max }$ in $\left(0, c_{0}\right)$ and precisely one local minimum $c_{\min }=-c_{\max }$ in $\left(-c_{0}, 0\right)$. These are determined as zeroes of $(22)$ :

$$
h^{\prime}\left(c_{\max }\right)=h^{\prime}\left(c_{\min }\right)=0,
$$

hence

$$
c_{\max }=-c_{\min }=1.840428142 \ldots, \quad \alpha_{\max }=h\left(c_{\max }\right)=1.343799725 \ldots
$$

Thanks to convexity and concavity we see that every number $\alpha \in\left(-\alpha_{\max }, \alpha_{\max }\right) \backslash\{0\}$ has precisely two preimages under $h$.

Remark 1. From the previous calculations we see that for the extremal parameter $\alpha_{\max }$ the boundary slope of the corresponding solution is $u^{\prime}(0)=-u^{\prime}(1)=G^{-1}\left(c_{\max } / 2\right)=1.586926484 \ldots$ 


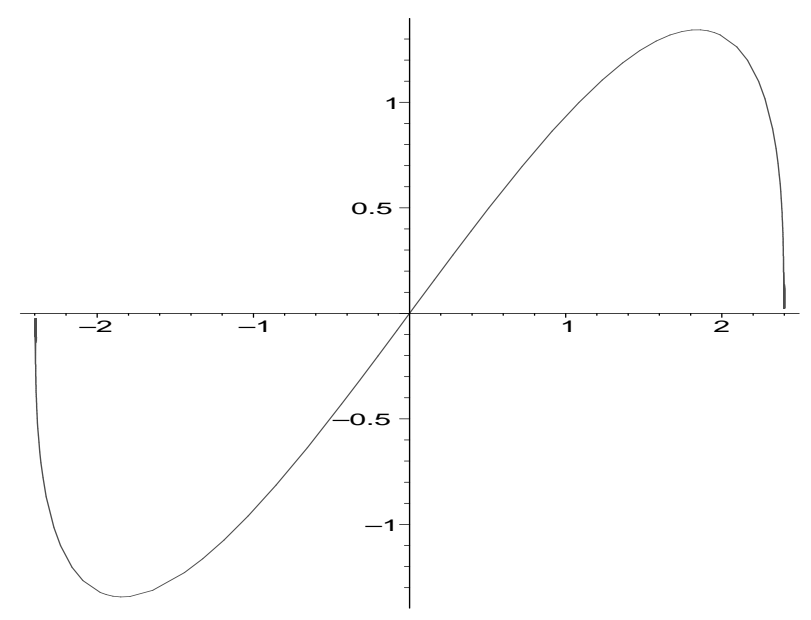

Figure 4: Admissible values of the boundary datum $\alpha$ plotted over the parameter $c$

\section{Energy of small and large solution}

We consider $\alpha$ such that $|\alpha| \leq \alpha_{\max }$ and determine the corresponding values of $c$ describing the solutions $u_{c}$ to (11) according to

$$
\alpha=\frac{c}{\sqrt[4]{1+G^{-1}\left(\frac{c}{2}\right)^{2}}} .
$$

Then the modified Willmore energy can be easily calculated as:

$$
\tilde{W}_{\alpha}\left(u_{c}\right)=c^{2}-4 \alpha \arctan G^{-1}\left(\frac{c}{2}\right) .
$$

In Figure 5 (left), we display the Willmore energy of the small and the large solution as a function of the boundary datum $\alpha$.
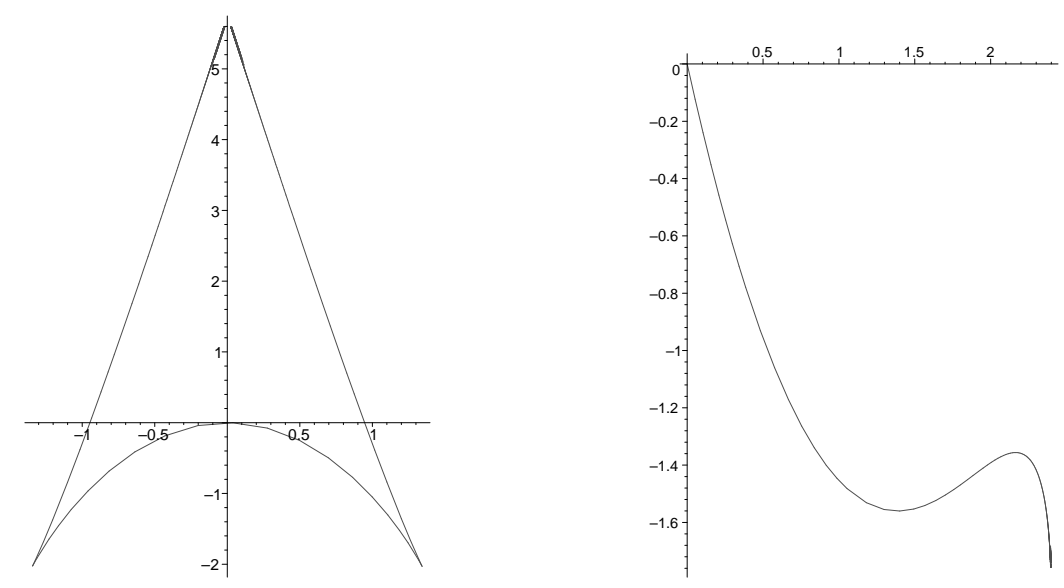

Figure 5: Energy of small and large solution of the Navier boundary value problem plotted over $\alpha$ (left) and energy of the functions $u_{c}$ for $\alpha=1.2$ plotted over $c \in\left[0, c_{0}\right)$ (right)

Looking at the Willmore energy (23) from a different point of view exhibits a somewhat unexpected feature: Let us consider some $\alpha<\alpha_{\max }$ relatively close to $\alpha_{\max }$ and keep this $\alpha$ fixed. Then it turns out that even in the family $\left\{u_{c}, c \in\left[0, c_{0}\right)\right\}$, the energy of the small solution with boundary datum $\alpha$ is not a global minimum. The infimum is approached by $\tilde{W}_{\alpha}\left(u_{c}\right)$ when $c \nearrow c_{0}$ and hence, it is not attained. See Figure 5 (right) for $\alpha=1.2$. 


\section{The Dirichlet boundary value problem}

The first part of Theorem 2 follows from (17):

$$
u^{\prime}(0)=-u^{\prime}(1)=G^{-1}\left(\frac{c}{2}\right)
$$

the strict monotonicity and continuity of $G^{-1}$ and from $G^{-1}\left(-c_{0} / 2, c_{0} / 2\right)=\mathbb{R}$. Next, let $v \in M_{\beta}$ be arbitrary. Recalling (20) we have

$$
\begin{aligned}
W & (v)-W(u) \\
& =\int_{0}^{1} \kappa_{v}(x)^{2} \sqrt{1+v^{\prime}(x)^{2}} d x-\int_{0}^{1} \kappa_{u}(x)^{2} \sqrt{1+u^{\prime}(x)^{2}} d x \\
& =\int_{0}^{1}\left|\kappa_{v}(x)\left(1+v^{\prime}(x)^{2}\right)^{\frac{1}{4}}-\kappa_{u}(x)\left(1+u^{\prime}(x)^{2}\right)^{\frac{1}{4}}\right|^{2} d x-2 c \int_{0}^{1} \frac{v^{\prime \prime}(x)}{\left(1+v^{\prime}(x)^{2}\right)^{\frac{5}{4}}} d x-2 c^{2} \\
& =\int_{0}^{1}\left|\kappa_{v}(x)\left(1+v^{\prime}(x)^{2}\right)^{\frac{1}{4}}-\kappa_{u}(x)\left(1+u^{\prime}(x)^{2}\right)^{\frac{1}{4}}\right|^{2} d x-2 c\left(G\left(v^{\prime}(1)\right)-G\left(v^{\prime}(0)\right)\right)-2 c^{2} \\
& =\int_{0}^{1}\left|\kappa_{v}(x)\left(1+v^{\prime}(x)^{2}\right)^{\frac{1}{4}}-\kappa_{u}(x)\left(1+u^{\prime}(x)^{2}\right)^{\frac{1}{4}}\right|^{2} d x
\end{aligned}
$$

since $G\left(v^{\prime}(1)\right)-G\left(v^{\prime}(0)\right)=-2 G(\beta)=-c$. From this we infer $W(v)>W(u)$ unless $v \equiv u$.

Open Problem 1. Can one show that suitable symmetry of Navier or Dirichlet boundary data implies symmetry of the solution?

\section{Nonsymmetric boundary value problems}

\section{Navier boundary conditions}

As a starting point we take the large explicit solution of the Navier problem (11) which we obtain formally when we let $c \nearrow c_{0}$, i.e. $\alpha \searrow 0$ :

$$
U_{0}(x)=\frac{2}{c_{0} \sqrt[4]{1+G^{-1}\left(\frac{c_{0}}{2}-c_{0} x\right)^{2}}} .
$$

One should observe that this solution is no longer smooth as a graph near $x=0$ and $x=1$, and for this reason, it was not included in Theorem 1 . However, as a curve in $\mathbb{R}^{2}$, it smoothly extends to the points $(0,0)$ and $(1,0)$ as a solution of the Navier problem with $\alpha=0$. Moreover, for its continuous odd extension - see Figure 6 -

$$
U_{0}(x)= \begin{cases}\frac{2}{c_{0} \sqrt[4]{1+G^{-1}\left(\frac{c_{0}}{2}-c_{0} x\right)^{2}}} & \text { if } x \in(0,1) \\ -\frac{2}{c_{0} \sqrt[4]{1+G^{-1}\left(\frac{c_{0}}{2}+c_{0} x\right)^{2}}} & \text { if } x \in(-1,0) \\ 0 & \text { if } x \in\{-1,0,1\}\end{cases}
$$

we can prove:

Lemma 7. The curve $[-1,1] \ni x \mapsto\left(x, U_{0}(x)\right) \in \mathbb{R}^{2}$ is a smooth curve and a critical point of the Willmore functional (7) with $\alpha=0$. 
Proof. Only the points $(-1,0),(0,0),(1,0)$ have to be considered. By symmetry it is enough to study the curve in a neighbourhood of $(0,0)$. Close to $x=0$, the function $x \mapsto U_{0}(x)$ is continuous and strictly monotone and hence has an inverse function

$$
y \mapsto V_{0}(y):=U_{0}^{-1}(y)
$$

for $y$ close to 0 , the explicit form of which ist given by

$$
V_{0}(y)= \begin{cases}\frac{1}{2}-\frac{1}{c_{0}} G\left(\sqrt{\left(\frac{2}{c_{0} y}\right)^{4}-1}\right) & \text { if } y>0 \\ -\frac{1}{2}+\frac{1}{c_{0}} G\left(\sqrt{\left(\frac{2}{c_{0} y}\right)^{4}-1}\right) & \text { if } y<0 \\ 0 & \text { if } y=0 .\end{cases}
$$

By continuity, $\lim _{x \rightarrow 0} U_{0}^{\prime}(x)=\infty$ and oddness, $V_{0} \in C^{1}$ is obvious. For $y$ close to 0 , we calculate

$$
V_{0}^{\prime}(y)=\frac{y^{2}}{\sqrt{\left(\frac{2}{c_{0}}\right)^{4}-y^{4}}},
$$

from which it is immediate that $V_{0} \in C^{\infty}$ in a neighbourhood of $y=0$.



Figure 6: Graph of $U_{0}$

In order to construct solutions to the Navier boundary value problem $(5,6)$ we select two points

$$
-1<x_{0}<x_{1}<1
$$

and take the line through

$$
\left(x_{0}, U_{0}\left(x_{0}\right)\right) \text { and }\left(x_{1}, U_{0}\left(x_{1}\right)\right)
$$

as the new $x$-axis, the new $y$-axis being orthogonal. In this coordinate system we obtain a solution of the Navier boundary value problem on an interval of length

$$
L\left(x_{0}, x_{1}\right):=\sqrt{\left(x_{1}-x_{0}\right)^{2}+\left(U_{0}\left(x_{1}\right)-U_{0}\left(x_{0}\right)\right)^{2}},
$$


which takes on 0-boundary values for the solution itself and

$$
\kappa_{0}\left(x_{0}\right) \text { and } \kappa_{0}\left(x_{1}\right)
$$

for the curvature. Here, the curvature function $\kappa_{0}$ according to $U_{0}$ is given by

$$
\kappa_{0}(x)=\left\{\begin{array}{ll}
-\frac{c_{0}}{\sqrt[4]{1+G^{-1}\left(\frac{c_{0}}{2}-c_{0} x\right)^{2}}} \cdot & \text { if } x \in(0,1) \\
\frac{c_{0}}{\sqrt[4]{1+G^{-1}\left(\frac{c_{0}}{2}+c_{0} x\right)^{2}}} \cdot & \text { if } x \in(-1,0) \\
0 & \text { if } x \in\{-1,0,1\}
\end{array}\right\}=-\frac{c_{0}^{2}}{2} U_{0}(x)
$$

In order to find out whether we have obtained a smooth graph, we have to check whether the angle between the new $x$-axis and the tangent vector of the curve (when passing from the left to the right) lies in $(-\pi / 2, \pi / 2)$, i.e. whether for $x \in\left[x_{0}, x_{1}\right]$, one has

$$
\left(x_{1}-x_{0}\right)+U_{0}^{\prime}(x)\left(U_{0}\left(x_{1}\right)-U_{0}\left(x_{0}\right)\right)>0 .
$$

If $x_{0}<0<x_{1}$, this is only a condition on those points $x$ with $U_{0}^{\prime}(x)<0$, because in this case $U_{0}\left(x_{1}\right)-U_{0}\left(x_{0}\right)>0$. In these regions, we know from Corollary 3 that $U_{0}^{\prime}$ is increasing resp. decreasing. If $x_{0}, x_{1}$ have the same sign, then $U_{0}^{\prime}$ is decreasing resp. increasing on the whole interval $\left[x_{0}, x_{1}\right]$. Hence, it is enough to check whether

$$
\left(x_{1}-x_{0}\right)+U_{0}^{\prime}\left(x_{j}\right)\left(U_{0}\left(x_{1}\right)-U_{0}\left(x_{0}\right)\right)>0, \quad j=0,1
$$

is satisfied. In view of (17) and (30) and since $U_{0}^{\prime}$ is an even function, this condition is equivalent to

$$
\left(x_{1}-x_{0}\right)+\frac{2}{c_{0}^{2}} G^{-1}\left(\frac{c_{0}}{2}-c_{0}\left|x_{j}\right|\right)\left(\kappa_{0}\left(x_{0}\right)-\kappa_{0}\left(x_{1}\right)\right)>0, \quad j=0,1 .
$$

We emphasize that for some suitable $\delta>0$, this condition is obviously satisfied for

$$
-\frac{1}{2}-\delta<x_{0}<x_{1}<\frac{1}{2}+\delta
$$

In what follows, we exclude the symmetric case $U_{0}\left(x_{0}\right)=U_{0}\left(x_{1}\right)$, for which we may refer to Theorems 1 and 2. For points $x_{0}, x_{1}$ subject to (31) the function

$$
x \mapsto \frac{x_{1}-x_{0}}{L\left(x_{0}, x_{1}\right)}\left(x-x_{0}\right)+\frac{U_{0}\left(x_{1}\right)-U_{0}\left(x_{0}\right)}{L\left(x_{0}, x_{1}\right)}\left(U_{0}(x)-U_{0}\left(x_{0}\right)\right), \quad x_{0} \leq x \leq x_{1}
$$

is strictly increasing and hence has an inverse function $\phi:\left[0, L\left(x_{0}, x_{1}\right)\right] \rightarrow\left[x_{0}, x_{1}\right]$. It is not difficult to verify that in the rotated coordinate system $\operatorname{graph}\left(U_{0 \mid\left[x_{0}, x_{1}\right]}\right)$ is given by

$$
\tilde{U}(y)=-\frac{L\left(x_{0}, x_{1}\right)}{U_{0}\left(x_{1}\right)-U_{0}\left(x_{0}\right)}\left(\phi(y)-x_{0}\right)+\frac{x_{1}-x_{0}}{U_{0}\left(x_{1}\right)-U_{0}\left(x_{0}\right)} y, \quad 0 \leq y \leq L\left(x_{0}, x_{1}\right) .
$$

Rescaling to the interval $[0,1]$ by setting $u(x)=\frac{1}{L\left(x_{0}, x_{1}\right)} \tilde{U}\left(L\left(x_{0}, x_{1}\right) x\right)$ we then find that

$$
u(x)=\frac{1}{U_{0}\left(x_{1}\right)-U_{0}\left(x_{0}\right)}\left(x_{0}-\phi\left(L\left(x_{0}, x_{1}\right) x\right)+\left(x_{1}-x_{0}\right) x\right), \quad x \in[0,1] .
$$





Figure 7: Admissible pairs of boundary curvatures in 0 and 1 for smooth graph solutions (left) and for Willmore curves (right) obtained by the described procedure and reflections

solves (5). In particular we have

$$
\kappa_{u}(0)=L\left(x_{0}, x_{1}\right) \kappa_{\tilde{U}}(0)=L\left(x_{0}, x_{1}\right) \kappa_{0}\left(x_{0}\right), \quad \kappa_{u}(1)=L\left(x_{0}, x_{1}\right) \kappa_{0}\left(x_{1}\right)
$$

so that, employing also reflections, we can realize the following curvatures on the interval $[0,1]$ as Navier boundary data for graph solutions of the Willmore equation

$$
\begin{aligned}
\mathcal{C}:= & \mathcal{C}_{0} \cup\left\{\left(a_{0}, a_{1}\right):\left(a_{1}, a_{0}\right) \in \mathcal{C}_{0}\right\} \\
& \cup\left\{\left(a_{0}, a_{1}\right):\left(-a_{0},-a_{1}\right) \in \mathcal{C}_{0}\right\} \cup\left\{\left(a_{0}, a_{1}\right):\left(-a_{1},-a_{0}\right) \in \mathcal{C}_{0}\right\}
\end{aligned}
$$

where

$$
\mathcal{C}_{0}:=\left\{\left(\kappa_{0}\left(x_{0}\right) L\left(x_{0}, x_{1}\right), \kappa_{0}\left(x_{1}\right) L\left(x_{0}, x_{1}\right)\right):-1<x_{0}<x_{1}<1 \text {; condition (32) is satisfied }\right\} .
$$

Theorem 3. Let $\mathcal{C} \subset \mathbb{R}^{2}$ be defined according to (34). Then for every $\left(\alpha_{0}, \alpha_{1}\right) \in-\mathcal{C}=\mathcal{C}$, the Navier boundary value problem

$$
\left\{\begin{array}{l}
\frac{1}{\sqrt{1+u^{\prime}(x)^{2}}} \frac{d}{d x}\left(\frac{\kappa^{\prime}(x)}{\sqrt{1+u^{\prime}(x)^{2}}}\right)+\frac{1}{2} \kappa^{3}(x)=0, \quad x \in(0,1), \\
u(0)=0, \quad u(1)=0, \quad \kappa(0)=-\alpha_{0}, \quad \kappa(1)=-\alpha_{1}
\end{array}\right.
$$

has a smooth (graph) solution.

The set $\mathcal{C}_{0}$ is plotted on the left of Fig. 7 , while those resulting pairs of curvatures, where we drop the condition on the curve being a graph is plotted on the right of this figure.

In Figure 8 we display the solution (left) obtained by the described procedure when choosing $x_{0}=-1 / 2$ and $x_{1}=1 / 2$. On the right its curvature is displayed.

In Figure 9 we display the solutions (left) obtained by the described procedure when choosing $x_{0}=0, x_{1}=0.7$ and $x_{0}=0, x_{1}=0.53 \ldots$ On the right their curvatures are shown.

Open Problem 2. We think that stable solutions are found among smooth graphs and that the set of admissible boundary curvatures, for which the Navier boundary value problem $(5,6)$ has a stable solution, coincides with $\mathcal{C}_{0}$. This conjecture is strongly supported by numerical evidence. 

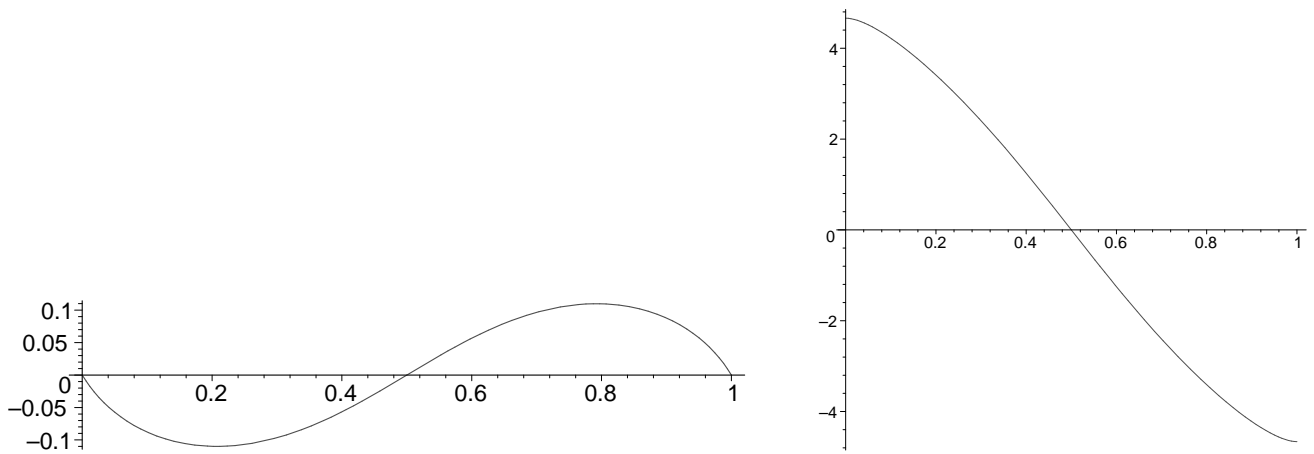

Figure 8: Graph (left) and curvature (right) of the solution obtained by choosing $x_{0}=-1 / 2$ and $x_{0}=1 / 2$


Figure 9: Graph (left) and curvature (right) of the solutions obtained by choosing $x_{0}=0, x_{1}=0.7$ and $x_{0}=0, x_{1}=0.53 \ldots$

Remark. As a Willmore curve, $U_{0}$ may be extended further by adding an odd reflection at the left or right end of the interval. Again, the above procedure may be applied to obtain Willmore curves, satisfying certain boundary conditions. These solutions, however, are no longer smooth graphs.

Since in this procedure, the length in (28) may become arbitrarily large while we achieve the same curvatures as above, the Navier boundary data may become arbitrarily large.

\section{Dirichlet boundary conditions}

Here, similar as in Theorem 2, we have an existence result without restrictions on the data. The proof, however, is not completely constructive.

Theorem 4. For every $\beta_{0}, \beta_{1} \in \mathbb{R}$, the Dirichlet boundary value problem

$$
\left\{\begin{array}{c}
\frac{1}{\sqrt{1+u^{\prime}(x)^{2}}} \frac{d}{d x}\left(\frac{\kappa^{\prime}(x)}{\sqrt{1+u^{\prime}(x)^{2}}}\right)+\frac{1}{2} \kappa^{3}(x)=0, \quad x \in(0,1), \\
u(0)=0, \quad u(1)=0, \quad u^{\prime}(0)=\beta_{0}, \quad u^{\prime}(1)=-\beta_{1}
\end{array}\right.
$$

for the Willmore equation has a smooth (graph) solution. 
Proof. The key observation is that the derivative of a solution is the arctan of the enclosed angle, which is invariant under rotation and under the rescaling

$$
u_{L}(x):=L u\left(\frac{1}{L} x\right),
$$

which transforms solutions of the Willmore equation into solutions and which we always employed in this section in order to rescale solutions to the unit interval.

In what follows, we develop the method from above introducing $x_{0}, x_{1}$ and corresponding rotated and rescaled parts of the graph of $U_{0}$ over the intersecting straight line from $\left(x_{0}, U_{0}\left(x_{0}\right)\right)$ to $\left(x_{1}, U_{0}\left(x_{1}\right)\right)$. For our purposes, it is convenient to extend $U_{0}$ oddly around the point $(1,0)$ to be defined on the interval $[-1,2]$.

We first look at the case where $\beta_{0}, \beta_{1} \geq 0$. In what follows we consider angles between the graph of $U_{0}$ and certain straight lines intersecting in $\left(x_{0}, U_{0}\left(x_{0}\right)\right)$ and $\left(x_{1}, U_{0}\left(x_{1}\right)\right)$ with the convention that in both points angles in $(-\pi, \pi)$ are counted positive, if the graph of $U_{0}$ is above the straight line in a right neighbourhood of $\left(x_{0}, U_{0}\left(x_{0}\right)\right)$ and a left neighbourhood of $\left(x_{1}, U_{0}\left(x_{1}\right)\right)$.

We proceed in two steps and start by proving that we can always solve for extreme values of the boundary data. The proof of all claims below is based upon continuity and the mean value theorem.

Claim 1. For given $\gamma_{0} \in[0, \pi / 2]$, we can find $-1 / 2<x_{0}<x_{1} \leq 1$ such that the angle is $\gamma_{0}$ in $\left(x_{0}, U_{0}\left(x_{0}\right)\right)$ and $\pi / 2$ in $\left(x_{1}, U_{0}\left(x_{1}\right)\right)$.

We start with $x_{1}=1$ and draw the straight line perpendicular to the graph of $U_{0}$, which intersects $U_{0}$ again in $x_{0}=0$ under the angle $\pi / 2$. Now we decrease $x_{1}$, consider the straight line through $\left(x_{1}, U_{0}\left(x_{1}\right)\right)$ perpendicular to the graph of $U_{0}$ and look for the largest intersection point $x_{0}<x_{1}$ with $U_{0}$. Before $x_{1}$ reaches $1 / 2$, this will no longer exist. So, there will be a limiting situation $\hat{x}_{0}<\hat{x}_{1}$ where the left angle is 0 and the right angle is $\pi / 2$. For the intermediate situations, all left angles between 0 and $\pi / 2$ are attained. See Figure 10.



Figure 10: Left angle $0 \ldots \pi / 2$, right angle $\pi / 2$

Claim 2. For given $\gamma_{0}, \gamma_{1} \in(0, \pi / 2)$, we can find $-1 / 2<x_{0}<x_{1}<3 / 2$ such that the angle is $\gamma_{0}$ in $\left(x_{0}, U_{0}\left(x_{0}\right)\right)$ and $\gamma_{1}$ in $\left(x_{1}, U_{0}\left(x_{1}\right)\right)$. 
Here, according to our first claim, we start with $x_{0}<x_{1}$ such that the angle on the left is $\gamma_{0}$ and on the right $\pi / 2$. Now we increase $x_{0}$ and keep the left angle $\gamma_{1}$ between the graph of $U_{0}$ and the intersecting straight line fixed. Since for large enough $x_{0}$, this straight line will become perpendicular, there is a limiting situation $\hat{x}_{0}<\hat{x}_{1}$, where the left angle is (still) $\gamma_{0}$ and where the angle in the intersection point on the right has decreased to 0. As before, the claim follows by virtue of the mean value theorem. See Figure 11.

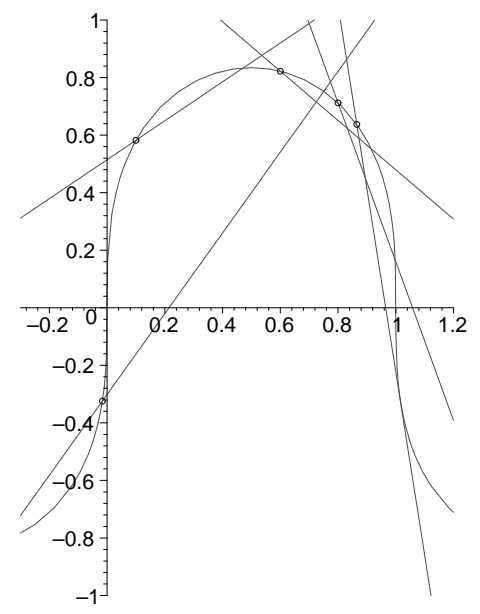

Figure 11: Left angle $\gamma_{0}$, right angle $0 \ldots \pi / 2$

We briefly comment on the remaining cases for $\beta_{0}, \beta_{1}$. When both are negative, we simply reflect the previously obtained solutions with respect to the $x$-axis.

If $\beta_{0}<0 \leq \beta_{1}$, we perform a similar procedure as above. We start within the first claim with $x_{0}=-1$ and subsequently obtain $-1 \leq x_{0}<x_{1} \leq 1$, where a prescribed negative angle is attained in the left intersection point and where we have a right angle in the intersection point on the right. See also Figure 10. Then, analogously to Claim 2, we keep the angle in the left point fixed and increase $x_{0}$. The angle in the right intersection point will finally decrease to 0 .

In the case that $\beta_{1}<0 \leq \beta_{0}$, the previous case and reflection with respect to the $u$-axis yield the claim.

Acknowledgement. The second author is grateful to G. Huisken (Max-Planck-Institute Golm) for suggesting to study boundary value problems for the Willmore equation. Moreover, we are grateful to the referee for a very careful reading of the manuscript.

\section{References}

[BK] M. Bauer, E. Kuwert, Existence of minimizing Willmore surface of prescribed genus, Int. Math. Res. Not. 2003, No.10, 553-576 (2003).

[DD] K. Deckelnick, G. Dziuk, Error analysis of a finite element method for the Willmore flow of graphs, Interfaces Free Bound. 8, 21-46 (2006).

[DKS] G. Dziuk, E. Kuwert, R. Schätzle, Evolution of elastic curves in $\mathbb{R}^{n}$ : Existence and computation, SIAM J. Math. Anal. 33, 1228-1245 (2002). 
[E] L. Euler, Opera Omnia, Ser. 1, 24, Zürich: Orell Füssli, 1952.

[KS1] E. Kuwert, R. Schätzle, The Willmore flow with small initial energy, J. Differ. Geom. 57, 409-441 (2001).

[KS2] E. Kuwert, R. Schätzle, Gradient flow for the Willmore functional, Commun. Anal. Geom. 10, 307-339 (2002).

[KS3] E. Kuwert, R. Schätzle, Removability of point singularities of Willmore surfaces, Annals Math. 160, 315-357 (2004).

[LS] J. Langer, D.A. Singer, The total squared curvature of closed curves, J. Differ. Geom. 20, 1-22 (1984).

[LI] A. Linnér, Explicit elastic curves, Ann. Global Anal. Geom. 16, 445-475 (1998).

[Nit] J.C.C. Nitsche, Boundary value problems for variational integrals involving surface curvatures, Quarterly Appl. Math. 51, 363-387 (1993).

[MS] U.F. Mayer, G. Simonett, A numerical scheme for axisymmetric solutions of curvaturedriven free boundary problems, with applications to the Willmore flow, Interfaces Free Bound. 4, 89-109 (2002).

[P] A. Polden, Curves and Surfaces of Least Total Curvature and Fourth-Order Flows, Ph.D. dissertation, University of Tübingen, 1996.

[Sn] L. Simon, Existence of surfaces minimizing the Willmore functional, Commun. Anal. Geom. 1, 281-326 (1993).

[St] G. Simonett, The Willmore flow near spheres, Differ. Integral Equ. 14, 1005-1014 (2001).

[W] T.J. Willmore, Total curvature in Riemannian geometry, Ellis Horwood Series in Mathematics and its Applications, Ellis Horwood Limited \& Halsted Press: Chichester, New York etc. (1982). 